\title{
FROM QUASIMODES TO RESONANCES
}

\author{
Siu-Hung Tang And Maciej Zworski
}

\section{Introduction}

Stefanov and Vodev [11], [12], obtained a remarkable result which says that for scattering by compactly supported perturbations in odd dimensional Euclidean space, existence of localized quasimodes implies existence of resonances rapidly converging to the real axis. The purpose of this note is to extend this result to all dimensions and to a wide class of non-compactly supported perturbations. Our method also gives lower bounds for the number of resonances in small neighbourhoods of the real axis. In fact, any quasi-mode construction will immediately provide a linear lower bound. A finer analysis of quasimodes should provide examples for which our main theorem gives the optimal lower bounds, $r^{n}$. A typical example is the Helmholtz resonator (see Fig.1) for which using the results of Babich-Buldyrev [1], Ralston [8], Lazutkin [4] and Popov [6] we obtain at least a linear growth of resonances in small neighbourhoods of the real axis. We could also consider metric perturbations - see [2] and references given there for construction of quasimodes for manifolds.

The argument of Stefanov and Vodev is an application of the PhragménLindelöf principle and of an a priori bound on the meromorphic continuation of the resolvent, $R(\lambda)$ :

$$
\|\chi R(\lambda) \chi\|_{\mathcal{H} \rightarrow \mathcal{H}} \leq C \exp \left(C|\lambda|^{N}\right), \lambda \notin \bigcup_{j=1}^{\infty} D\left(\lambda_{j},\left\langle\lambda_{j}\right\rangle^{-M}\right), \quad \chi \in C_{0}^{\infty}\left(\mathbb{R}^{n}\right),
$$

where $\lambda_{j}$ are resonances, that is, the poles of $R(\lambda)$, and $M, N$ are sufficiently large depending on the perturbation. The bound (1) was obtained by the second author [14] for obstacle scattering using the methods developed by Melrose [5] (see Proposition 5.2 of [11] and the remark on page 680 there) and was extended to more general perturbations using the methods of [13] in [11],[12].

In this paper we work in the semi-classical setting of "black box scattering" introduced by Sjöstrand and the second author in [10] and then extended by Sjöstrand in [9]. In the modification of the argument of Stefanov and Vodev we replace the Phragmén-Lindelöf principle by a local application of the maximum principle adapted to the semi-classical setting. The global bound (1) is replaced

Received January 15, 1998.

The research of the first author was supported in part by the Purdue Research Foundation and that of the second author by the National Science and Engineering Research Council of Canada. 


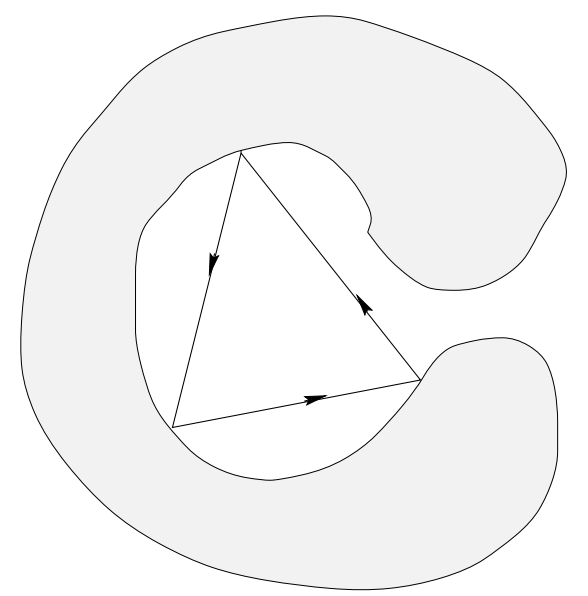

Figure 1. A domain with elliptic trapped rays: "The Helmholtz resonator".

by a local bound given in Lemma 1 below and coming essentially from the recent work of Sjöstrand on the local trace formula for resonances [9].

It is interesting to note that the global trace formula for resonances (established in successive generality by Bardos-Guillot-Ralston, Melrose, SjöstrandZworski and Sá Barreto-Zworski - see [9],[15] and references given there) can be proved using the minimum modulus theorem as in the proof of (1) - see [3],[15], while for the more generally valid local formula of [9], the local estimates of the type used here are needed.

Finally we remark that the results of Stefanov and Vodev, [11],[12], and the more recent results of Popov and Vodev [7] are also concerned with situations in which one cannot construct quasi-modes in the standard sense. By proceeding as in their papers and using the methods of this paper one could generalize their results to even dimensions and to suitable non-compactly supported perturbations.

\section{Preliminaries}

We will work in the semi-classical category and we review here the framework of "black box" scattering from [9]. Thus, let $\mathcal{H}$ be a complex Hilbert space with an orthogonal decomposition

$$
\mathcal{H}=\mathcal{H}_{R_{0}} \oplus L^{2}\left(\mathbb{R}^{n} \backslash B\left(0, R_{0}\right)\right),
$$

where $R_{0}>0$ is fixed and $B(x, R)=\left\{y \in \mathbb{R}^{n}:|x-y|<R\right\}$. The corresponding orthogonal projections are denoted by $\left.u\right|_{B\left(0, R_{0}\right)}$ and $\left.u\right|_{\mathbb{R}^{n} \backslash B\left(0, R_{0}\right)}$ or by $\mathbb{1}_{B\left(0, R_{0}\right)} u$ and $\mathbb{1}_{\mathbb{R}^{n} \backslash B\left(0, R_{0}\right)} u$ respectively, where $u \in \mathcal{H}$.

For each $h \in\left(0, h_{0}\right]$, we have an unbounded self-adjoint operator

$$
P(h): \mathcal{H} \longrightarrow \mathcal{H}
$$


with domain $\mathcal{D}$, independent of $h$, which satisfies

$$
\mathbb{1}_{\mathbb{R}^{n} \backslash B\left(0, R_{0}\right)} \mathcal{D}=H^{2}\left(\mathbb{R}^{n} \backslash B\left(0, R_{0}\right)\right)
$$

uniformly with respect to $h$ in the following sense:

Equip $H^{2}\left(\mathbb{R}^{n} \backslash B\left(0, R_{0}\right)\right)$ with the norm $\left\|\langle h D\rangle^{2} u\right\|_{L^{2}}$, where

$$
\langle h D\rangle=\left(1+(h D)^{2}\right)^{1 / 2},(h D)^{2}=\sum_{j=1}^{n}\left(h D_{x_{j}}\right)^{2},
$$

and equip $\mathcal{D}$ with the norm $\|(i+P(h)) u\|_{\mathcal{H}}$, then we require the projection map

$$
\mathbb{1}_{\mathbb{R}^{n} \backslash B\left(0, R_{0}\right)}: \mathcal{D} \longrightarrow H^{2}\left(\mathbb{R}^{n} \backslash B\left(0, R_{0}\right)\right)
$$

to be bounded uniformly with respect to $h$ and to have a uniformly bounded right inverse.

We also assume $P(h)$ to satisfy the following conditions:

$$
\begin{gathered}
\mathbb{1}_{B\left(0, R_{0}\right)}(P(h)+i)^{-1}: \mathcal{H} \longrightarrow \mathcal{H}_{R_{0}} \text { is compact, } \\
\mathbb{1}_{\mathbb{R}^{n} \backslash B\left(0, R_{0}\right)} P(h) u=Q(h)\left(\left.u\right|_{\mathbb{R}^{n} \backslash B\left(0, R_{0}\right)}\right), \quad \text { for } u \in \mathcal{D},
\end{gathered}
$$

where $Q(h)$ is a formally self-adjoint operator on $L^{2}\left(\mathbb{R}^{n}\right)$ given by

$$
Q(h) v=\sum_{|\alpha| \leq 2} a_{\alpha}(x ; h)\left(h D_{x}\right)^{\alpha} v \text { for } v \in C_{0}^{\infty}\left(\mathbb{R}^{n}\right)
$$

such that

(a) $a_{\alpha}(x ; h)=a_{\alpha}(x)$ is independent of $h$ for $|\alpha|=2$,

(b) $a_{\alpha}(x ; h) \in C_{b}^{\infty}\left(\mathbb{R}^{n}\right)$ are uniformly bounded with respect to $h$, here $C_{b}^{\infty}\left(\mathbb{R}^{n}\right)$ denotes the space of $C^{\infty}$ functions on $\mathbb{R}^{n}$ with bounded derivatives of all orders,

(c) $\sum_{|\alpha|=2} a_{\alpha}(x ; h) \xi^{\alpha} \geq(1 / c)|\xi|^{2}, \quad \forall \xi \in \mathbb{R}^{n}$, for some constant $c>0$,

(d) $\sum_{|\alpha| \leq 2} a_{\alpha}(x ; h) \xi^{\alpha} \longrightarrow \xi^{2}$ uniformly with respect to $h$ as $|x| \rightarrow \infty$,

(3) there exist $\theta \in[0, \pi), \epsilon>0$ and $R \geq R_{0}$ such that the coefficients $a_{\alpha}(x ; h)$ of $Q(h)$ extend holomorphically in $x$ to

$$
\left\{r \omega: \omega \in \mathbb{C}^{n}, \operatorname{dist}\left(\omega, S^{n}\right)<\epsilon, r \in \mathbb{C},|r|>R, \arg r \in\left[-\epsilon, \theta_{0}+\epsilon\right)\right\}
$$

with $\sum_{|\alpha| \leq 2} a_{\alpha}(x ; h) \xi^{\alpha} \longrightarrow \xi^{2}$ uniformly with respect to $h$ as $|x| \rightarrow \infty$ remains valid in this larger set of $x$,

(4) We use $P(h)$ to construct a self-adjoint operator $P^{\sharp}(h)$ on

$$
\mathcal{H}^{\sharp}=\mathcal{H}_{R_{0}} \oplus L^{2}\left(M \backslash B\left(0, R_{0}\right)\right)
$$

as in [10] where $M=(\mathbb{R} / R \mathbb{Z})^{n}$ for some $R \gg R_{0}$. Let $N\left(P^{\sharp}(h), I\right)$ denote the number of eigenvalues of $P^{\sharp}(h)$ in the interval $I$, we assume

$$
N\left(P^{\sharp}(h),[-\lambda, \lambda]\right)=\mathcal{O}\left(\left(\lambda / h^{2}\right)^{n^{\sharp} / 2}\right), \text { for } \lambda \geq 1
$$


for some number $n^{\sharp} \geq n$.

Under the above assumptions on $P(h)$ the resonances close to the the real axis can be defined by the method of complex scaling (see [9]). They coincide with the poles the meromorphic continuation of the resolvent $(P(h)-z)^{-1}$ from $\operatorname{Im} z>0$ to a conic neighbourhood of the positive half axis in the lower half plane. The set of resonances of $P(h)$ will be denoted by $\operatorname{Res} P(h)$ and we include them with their multiplicity.

For the more standard case of an operator $P$ which is independent of $h$ (such as, for instance, a compact perturbation of the Laplacian) we only need to verify the assumptions for $h=1$ and consider $P(h)=h^{2} P$. Then the poles of the meromorphic continuation of $R(\lambda)=\left(P-\lambda^{2}\right)^{-1}$ from the upper half plane to a conic neighbourhood of the real axis are related to the resonances of $P(h)$ : $\lambda^{2}=h^{-2} z$ for the poles, $z$, of the continuation of $(P(h)-z)^{-1}$ from $\operatorname{Im} z>0$. We will denote the set of poles of $R(\lambda)$, that is, of scattering poles, by $\mathcal{R}_{P}$.

\section{Statement of the results}

In the framework reviewed in the previous section we can now state our main result:

Theorem. Suppose $P(h)$ satisfy the assumptions stated in Sec.2. If for all $h \leq h_{0}$, there exist a finite index set $\Lambda(h)$, sequences $\left\{E_{j}(h)\right\}_{j \in \Lambda(h)} \subset\left[E_{0}-h\right.$, $\left.E_{0}+h\right]$ and $\left\{u_{j}(h)\right\}_{j \in \Lambda(h)} \subset \mathcal{H}$ with $\left\|u_{j}(h)\right\|_{\mathcal{H}}=1$ such that

- $\left|E_{i}(h)-E_{j}(h)\right| \geq 15 h^{k}$ for all $i, j \in \Lambda(h)$ with $i \neq j$ and for some fixed constant $k>1$

- $\left\|\left(P(h)-E_{j}(h)\right) u_{j}(h)\right\|_{\mathcal{H}}=R(h) \quad$ for all $j \in \Lambda(h)$ with some positive function $R(h)=\mathcal{O}\left(h^{\infty}\right)$

- $\operatorname{supp}\left(1_{\mathbb{R}^{n} \backslash B\left(0, R_{0}\right)} u j(h)\right) \subset K \Subset \mathbb{R}^{n}$ for all $j \in \Lambda(h)$

Then, for any positive function $S(h)$ satisfying $S(h) \gg R(h)$ and $D e^{-D / h} \leq$ $S(h)=\mathcal{O}\left(h^{\infty}\right)$ for some constant $D>0$, there exists $h(S)>0$ such that for all $h<h(S)$, we have

$$
\left(\left[E_{j}(h)-6 h^{k}, E_{j}(h)+6 h^{k}\right]+i\left[-S(h) h^{-n^{\sharp}-1}, 0\right]\right) \cap \operatorname{Res} P(h) \neq \phi \quad \forall j \in \Lambda(h),
$$

where $\operatorname{Res} P(h)$ denotes the set of resonances of $P(h)$.

In other words the existence of well separated quasi-modes gives the existence of many resonances close to the quasi-modes. We also remark ${ }^{1}$ that we can replace $h^{k}$ in the Theorem above by a positive function $w(h)=\mathcal{O}\left(h^{\infty}\right)$. The proof remains essentially unchanged provided that we require that $S(h)$ satisfies

$$
\frac{w^{2}(h)}{S(h) h^{-n^{\sharp}-1}} \rightarrow \infty \text { as } h \rightarrow 0 .
$$

Hence we can say that the resonances are very close to the quasimodes.

\footnotetext{
${ }^{1}$ We owe this remark to a conversation with Shu Nakamura.
} 
We will prove the theorem in the next section. Now, let us translate it to the classical setting where we can obtain lower bounds for the counting function of resonances in small neighbourhoods of the real axis.

Corollary. Let $P: \mathcal{H} \longrightarrow \mathcal{H}$ be an unbounded self-adjoint operator which satisfies the assumptions stated in Sec.2 with $h=1$. Put $P(h)=h^{2} P$ for $h \leq h_{0}$ and assume $P(h)$ satisfy the conditions in Theorem above with $|\Lambda(h)| \geq$ $\max \left(1, A h^{-m+1}\right)$ for some positive constants $A$ and $m \geq 1$. Then there exists a positive function, defined on $\mathbb{R}_{+}, T(x)=\mathcal{O}\left(x^{-\infty}\right)$ as $x \rightarrow+\infty$ such that for some constant $C=C(T)>0$, we have

$$
N(r)=\sharp\left\{\lambda_{j} \in \mathcal{R}_{P}: \operatorname{Re} \lambda_{j} \leq r, 0<-\operatorname{Im} \lambda_{j}<T\left(\left|\lambda_{j}\right|\right)\right\} \geq r^{m} / C .
$$

Proof. We first recall that $z \in \operatorname{Res} P(h) \Leftrightarrow h^{-1} \sqrt{z} \in \mathcal{R}_{P}$. Now from Theorem above, we have

$$
\left(\left[E_{j}(h)-6 h^{k}, E_{j}(h)+6 h^{k}\right]+i\left[-S(h) h^{-n^{\sharp}-1}, 0\right]\right) \cap \operatorname{Res} P(h) \neq \phi \forall j \in \Lambda(h) .
$$

The separation condition $\left|E_{i}(h)-E_{j}(h)\right| \geq 15 h^{k}$ for $i \neq j$ in the assumption of the Theorem then implies that

$$
\sharp\left\{\left(\left[E_{0}-h, E_{0}+h\right]+i\left[-S(h) h^{-n^{\sharp}-1}, 0\right]\right) \cap \operatorname{Res} P(h) \geq|\Lambda(h)| \geq A h^{-m+1} .\right.
$$

By scaling, we may assume $E_{0}=1$. Set

$$
\tilde{\Omega}(h)=\sqrt{[1-h, 1+h]+i\left[-S(h) h^{-n^{\sharp}-1}, 0\right]} .
$$

Put $T(x)=S\left(x^{-1}\right) x^{n^{\sharp}+1}=\mathcal{O}\left(x^{-\infty}\right)$ so that all boxes of the form $h^{-1} \tilde{\Omega}(h)$ lie above the curve $-\operatorname{Im} \lambda=T(|\lambda|)$ for $|\lambda|$ large enough. Now for $r \gg 1$ and $1 \ll l \leq r$, we have (the constant $C$ below may vary from line to line)

$$
\begin{aligned}
\sharp\left\{\left\{\lambda_{j} \in \mathcal{R}_{P}: \operatorname{Re} \lambda_{j} \leq r, 0<-\operatorname{Im} \lambda_{j}<T\left(\left|\lambda_{j}\right|\right)\right\} \cap[l, l+1]\right\} & \geq\left|\Lambda\left(l^{-1}\right)\right| / C \\
& \geq l^{m-1} / C .
\end{aligned}
$$

Thus,

$$
N(r) \geq \sum_{l=1}^{r-1} l^{m-1} / C \geq \frac{1}{C} \int_{0}^{r-1} x^{m-1} d x \geq r^{m} / C,
$$

which gives the corollary.

As remarked in Sect.1, any construction of quasimodes in the classical setting (see [2], [6], [8] and references given there) will give the assumptions of the theorem with any $E_{0}>0$ and $m=1$. The corollary then gives a linear lower bound. If one succeeds in constructing many quasimodes which are well separated, better bounds will result as the assumptions of Theorem above should then be satisfied with a larger $m$. Also, we can take $T(x)$ above to be $\mathcal{O}\left(e^{-c x}\right)$ if we can construct quasimodes with $R(h)=\mathcal{O}\left(e^{-c / h}\right)$ 


\section{Proof of the main theorem}

To prove Theorem of Sect. 3 we shall need the following two lemmas

Lemma 1. For any simply connected compact set $\tilde{\Omega} \subset S_{\theta}$ where

$$
S_{\theta}=\{z \in \mathbb{C}: \operatorname{Max}(-\pi, 2 \theta-2 \pi)<-\arg z<2 \theta\}
$$

( $\theta$ as in condition 3 of $P(h)$ ) and positive function $g(h) \ll 1$ defined on $0<h<$ $h_{0}$, there exist constants $A=A(\tilde{\Omega})>0$ and $h_{1}$ with $0<h_{1}<h_{0}$ such that

$$
\left\|\chi(P(h)-z)^{-1} \chi\right\|_{\mathcal{H} \rightarrow \mathcal{H}} \leq A e^{A h^{-n^{\sharp}} \log \frac{1}{g(h)}} \quad \forall z \in \tilde{\Omega} \backslash \bigcup_{z_{j} \in \operatorname{Res} P(h) \cap \tilde{\Omega}} D\left(z_{j}, g(h)\right)
$$

for $h<h_{1}$, where $\chi \in C_{0}^{\infty}\left(\mathbb{R}^{n}\right)$ with $\chi=1$ near $B\left(0, R_{0}\right)$, and $D\left(z_{j}, g(h)\right)=$ $\left\{z \in \mathbb{C},\left|z_{j}-z\right| \leq g(h)\right\}$.

Lemma 2. Let $\left\{h_{j}\right\}_{j \geq 1} \subset \mathbb{R}_{+}$be a sequence such that $h_{j} \rightarrow 0$ as $j \rightarrow \infty$. Suppose $F(z, h)$ is a holomorphic function defined in a neighborhood of

$$
\Omega(h)=\left(\left[E(h)-5 h^{k}, E(h)+5 h^{k}\right]+i\left[-S(h) h^{-n^{\sharp}-1}, S(h)\right]\right)
$$

for $h \in\left\{h_{j}\right\}_{j \geq 1}$ and $E(h) \in \mathbb{R}$, where $S(h)$ is as in Theorem. If $F(z, h)$ satisfy

$$
\begin{gathered}
|F(z, h)| \leq A e^{A h^{-n^{\sharp}} \log \frac{1}{h S(h)}}, \text { on } \Omega(h) \\
|F(z, h)| \leq 1 / \operatorname{Im} z, \text { on } \Omega(h) \cap\{\operatorname{Im} z>0\} .
\end{gathered}
$$

Then there exists $h(S)>0, B>0$ such that

$$
|F(z, h)| \leq B / S(h), \quad \forall z \in\left[E(h)-h^{k}, E(h)+h^{k}\right]
$$

when $h \in\left\{h_{j}\right\}_{j \geq 1}$ and $h \leq h(S)$.

Assuming Lemma 1 and Lemma 2 for the moment, we proceed to the

Proof of Theorem. Assume that the conclusion in our theorem is not true, that is, there exists a sequence $\left\{h_{j}\right\}_{j \geq 1} \subset \mathbb{R}_{+}$with $h_{j} \rightarrow 0$ as $j \rightarrow \infty$ such that

$$
\begin{array}{r}
\left(\left[E_{l}\left(h_{j}\right)-6 h_{j}^{k}, E_{l}\left(h_{j}\right)+6 h_{j}^{k}\right]+i\left[-S\left(h_{j}\right) h_{j}^{-n^{\sharp}-1}, 0\right]\right) \cap \operatorname{Res} P\left(h_{j}\right)=\phi, \\
\quad \text { for some } l_{j} \in \Lambda\left(h_{j}\right)
\end{array}
$$

Now, fix $\chi \in C_{0}^{\infty}\left(\mathbb{R}^{n}\right)$ with $\chi=1$ near $K$. Take any $h \in\left\{h_{j}\right\}_{j \geq 1}$ such that $h<h(S)(h(S)$ as in Lemma 2$)$ and $l=l_{j}$. We have

(1) $\chi(P(h)-z)^{-1} \chi$ defines a holomorphic (operator-valued) function on

$$
\left[E_{l}(h)-6 h^{k}, E_{l}(h)+6 h^{k}\right]+i\left[-S(h) h^{-n^{\sharp}-1}, S(h)\right] \text { for some } l \in \Lambda(h) .
$$


(2) By Lemma 1 (choosing $g(h)=h S(h)$ ), we have

$$
\left\|\chi(P(h)-z)^{-1} \chi\right\|_{\mathcal{H} \rightarrow \mathcal{H}} \leq A e^{A\left(\log \frac{1}{h S(h)}\right) h^{-n^{\sharp}}}, \text { for } z \in \Omega(h),
$$

$(\Omega(h)$ as in Lemma 2$)$.

(3) For $z \in \Omega(h) \cap\{\operatorname{Im} z>0\}$, we have the following standard estimate (as $P(h)$ is self-adjoint)

$$
\left\|\chi(P(h)-z)^{-1} \chi\right\|_{\mathcal{H} \rightarrow \mathcal{H}} \leq 1 / \operatorname{Im} z .
$$

Combining Lemma 2 and 1, 2, 3 above, we have

$$
\left\|\chi(P(h)-z)^{-1} \chi\right\|_{\mathcal{H} \rightarrow \mathcal{H}} \leq \frac{B}{S(h)} \text { for } z \in\left[E_{l}(h)-h^{k}, E_{l}(h)+h^{k}\right] .
$$

Hence,

$$
\begin{gathered}
\left\|u_{l}(h)\right\|_{\mathcal{H}}=\left\|\left(P(h)-E_{l}(h)\right)^{-1}\left(P(h)-E_{l}(h)\right) u_{l}(h)\right\|_{\mathcal{H}} \\
\leq\left\|\chi\left(P(h)-E_{l}(h)\right)^{-1} \chi\right\|_{\mathcal{H} \rightarrow \mathcal{H}}\left\|\left(P(h)-E_{l}(h)\right) u_{l}(h)\right\|_{\mathcal{H}} \\
\leq \frac{B}{S(h)} R(h) \ll 1
\end{gathered}
$$

contradicting $\left\|u_{l}(h)\right\|_{\mathcal{H}}=1$ for all $h \leq h_{0}$ and $l \in \Lambda(h)$.

It remains now to prove Lemmas 1 and 2.

Proof of Lemma 1. The essential step in the proof of this lemma comes almost directly from [9]. Here, we shall only give indications of how to extract it from there. To conform with the notation of that paper, we will not write the dependence of $h$ explicitly in the following.

The estimate for the cut-off resolvent comes from estimates for the resolvent of the scaled operator. We recall from [10] and [9] that the operator $P$ satisfying the assumptions above is deformed to a non-self-adjoint operator $P_{\theta}$ acting on a Hilbert space $\mathcal{H}_{\theta}$ and with domain $\mathcal{D}_{\theta} \subset \mathcal{H}_{\theta}$. The operator $P_{\theta}-z$ is Fredholm for $z \in S_{\theta}$ and its complex eigenvalues, which are independent of $\theta$, are the resonances of $P$. A more precise statement is given in 1 below.

(1) From the argument in the proof of Lemma 3.5 in [10], we have

$$
\chi(P-z)^{-1} \chi=\chi\left(P_{\theta}-z\right)^{-1} \chi \quad \text { for } z \in S_{\theta}
$$

Hence, we are reduced to estimating $\left\|\left(P_{\theta}-z\right)^{-1}\right\|_{\mathcal{H}_{\theta} \rightarrow \mathcal{D}_{\theta}}$ for $z \in \tilde{\Omega}$.

(2) From (6.11), (6.12) in [9], we have

$$
\exists K \in L\left(\mathcal{H}_{\theta}, \mathcal{H}_{\theta}\right) \text { with }\|K\|_{\mathcal{H}_{\theta} \rightarrow \mathcal{H}_{\theta}}=\mathcal{O}(1) \text { and } \operatorname{rank} K=\mathcal{O}\left(h^{-n^{\sharp}}\right)
$$

such that

$$
\begin{gathered}
\left(P_{\theta}+K-z\right)^{-1} \text { is well defined and is } \mathcal{O}(1): \mathcal{H}_{\theta} \rightarrow \mathcal{D}_{\theta} \quad \forall z \in \Omega \\
\text { where } S_{\theta} \supset \Omega \ni \tilde{\Omega} \text { such that } \operatorname{Res} P(h) \cap \Omega=\operatorname{Res} P(h) \cap \tilde{\Omega} .
\end{gathered}
$$


(3) Using $K$ above, we can construct an invertible operator (see page 404 in [9])

$$
\mathcal{P}(z)=\left(\begin{array}{cc}
P_{\theta}-z & R_{-} \\
R_{+} & 0
\end{array}\right): \mathcal{D} \oplus \mathbb{C}^{N} \longrightarrow \mathcal{H} \oplus \mathbb{C}^{N}, z \in \Omega
$$

where $N=\operatorname{rank} K=\mathcal{O}\left(h^{-n^{\sharp}}\right)$ (note that the subscript $\theta$ in $\mathcal{D}_{\theta}$ and $\mathcal{H}_{\theta}$ is dropped as they are identified with $\mathcal{D}$ and $\left.\mathcal{H}\right)$. Its inverse

$$
\mathcal{E}(z)=\left(\begin{array}{cl}
E(z) & E_{+}(z) \\
E_{-}(z) & E_{-+}(z)
\end{array}\right): \mathcal{H} \oplus \mathbb{C}^{N} \longrightarrow \mathcal{D} \oplus \mathbb{C}^{N}, \quad z \in \Omega
$$

satisfies $\|\mathcal{E}(z)\|_{\mathcal{H} \oplus \mathbb{C}^{N} \rightarrow \mathcal{D} \oplus \mathbb{C}^{N}}=\mathcal{O}(1)$. Hence, the same is true for

$$
\begin{aligned}
\|E(z)\|_{\mathcal{H} \rightarrow \mathcal{D}}, & \left\|E_{+}(z)\right\|_{\mathbb{C}^{N} \rightarrow \mathcal{D}}, \\
\left\|E_{-}(z)\right\|_{\mathcal{H} \rightarrow \mathbb{C}^{N}}, & \left\|E_{-+}(z)\right\|_{\mathbb{C}^{N} \rightarrow \mathbb{C}^{N}},
\end{aligned}
$$

see $(6.22)$ in $[9]$.

(4) For $z \in \Omega \backslash \operatorname{Res} P$, we have (see (8.11) in [9])

$$
\left(P_{\theta}-z\right)^{-1}=E(z)-E_{+}(z) E_{-+}^{-1}(z) E_{-}(z) .
$$

Hence,

$$
\begin{gathered}
\left\|\left(P_{\theta}-z\right)^{-1}\right\|_{\mathcal{H} \rightarrow \mathcal{D}} \\
\leq\|E(z)\|_{\mathcal{H} \rightarrow \mathcal{D}}+\left\|E_{+}(z)\right\|_{\mathbb{C}^{N} \rightarrow \mathcal{D}}\left\|E_{-+}^{-1}(z)\right\|_{\mathbb{C}^{N} \rightarrow \mathbb{C}^{N}}\left\|E_{-}(z)\right\|_{\mathcal{H} \rightarrow \mathbb{C}^{N}} .
\end{gathered}
$$

From 3 , we see that it suffices to estimate $\left\|E_{-+}^{-1}(z)\right\|_{\mathbb{C}^{N} \rightarrow \mathbb{C}^{N}}$.

(5) Now,

$$
E_{-+}^{-1}(z)=\frac{\widetilde{E}_{-+}(z)}{\operatorname{det} E_{-+}(z)},
$$

where $\widetilde{E}$ is the minor of $E$. Using $\left\|E_{-+}(z)\right\|_{\mathbb{C}^{N} \rightarrow \mathbb{C}^{N}}=\mathcal{O}(1), N=$ $\mathcal{O}\left(h^{-n^{\sharp}}\right)$ and the following inequalites for a $N \times N$ matrix $A=\left(a_{i j}\right)$

$$
\|A\| \leq N \operatorname{Sup}\left|a_{i j}\right|,|\operatorname{det} A| \leq\|A\|^{N} .
$$

We have

$$
\left\|\widetilde{E}_{-+}(z)\right\| \leq B h^{-n^{\sharp}} e^{B h^{-n^{\sharp}}}
$$

for some constant $B=B(\Omega)>0$.

(6) It remains to estimate $\left|\operatorname{det} E_{-+}(z)\right|$ from below away from $z \in \operatorname{Res} P \cap \Omega$. Write

$$
\begin{gathered}
D(z ; h)=\operatorname{det} E_{-+}(z), D_{w}(z ; h)=\prod_{z_{j} \in \operatorname{Res} P \cap \Omega}\left(z-z_{j}\right) \\
D(z ; h)=G(z ; h) D_{w}(z ; h) .
\end{gathered}
$$


From the statement below (8.42) in [9], we have

$$
|G(z ; h)| \geq e^{-C h^{-n^{\sharp}}} \text { in } \tilde{\Omega} \text {. }
$$

Hence, for all $z \in \tilde{\Omega} \backslash \bigcup_{z_{j} \in \operatorname{Res} P(h) \cap \tilde{\Omega}} D\left(z_{j}, g(h)\right)$, we have

$$
\begin{aligned}
|D(z ; h)| & =|G(z ; h)|\left|D_{w}(z ; h)\right| \\
& \geq e^{-C h^{-n^{\sharp}}}(g(h))^{\mathcal{O}\left(h^{-n^{\sharp}}\right)} \\
& \geq \tilde{C} e^{-\tilde{C}\left(\log \frac{1}{g(h)}\right) h^{-n^{\sharp}}} \text { for some constant } \tilde{C}=\tilde{C}(\tilde{\Omega})>0 .
\end{aligned}
$$

Combining 4, 5, 6 we have

$$
\left\|\left(P_{\theta}-z\right)^{-1}\right\|_{\mathcal{H}_{\theta} \rightarrow \mathcal{D}_{\theta}} \leq A e^{A h^{-n^{\sharp}} \log \frac{1}{g(h)}}, \forall z \in \tilde{\Omega} \backslash \bigcup_{z_{j} \in \operatorname{Res} P(h) \cap \tilde{\Omega}} D\left(z_{j}, g(h)\right)
$$

for $h$ sufficiently small.

Finally, from 1, we have

$$
\begin{gathered}
\left\|\chi(P-z)^{-1} \chi\right\|_{\mathcal{H} \rightarrow \mathcal{H}}=\left\|\chi\left(P_{\theta}-z\right)^{-1} \chi\right\|_{\mathcal{H} \rightarrow \mathcal{H}} \leq\left\|\left(P_{\theta}-z\right)^{-1}\right\|_{\mathcal{H}_{\theta} \rightarrow \mathcal{H}_{\theta}} \\
\leq A e^{A h^{-n^{\sharp}} \log \frac{1}{g(h)}}, \quad \forall z \in \tilde{\Omega} \backslash \bigcup_{z_{j} \in \operatorname{Res} P(h) \cap \tilde{\Omega}} D\left(z_{j}, g(h)\right),
\end{gathered}
$$

for $h$ sufficiently small which concludes the proof.

Proof of Lemma 2. We will apply the maximum principle to the product of $F(z, h)$ with a suitable auxillary function. So, we divide the proof into two steps. The first is a construction of auxillary functions. We claim that there exists $\tilde{h}>0$ such that for any $h \in\left\{h_{j}\right\}_{j \geq 1}, h<\tilde{h}$, we can find a function $f(z, h)$ defined in a neighborhood of $\Omega(h)$ satisfying the following

(1) $f(z, h)$ is holomorphic in $\Omega(h)$

(2) $|f(z, h)| \leq e$ in $\Omega(h)$

(3) $|f(z, h)| \geq \frac{1}{2}$ for $z \in\left[E(h)-h^{k}, E(h)+h^{k}\right]$

(4) $|f(z, h)| \leq C e^{-C \frac{h^{2 k}}{\alpha^{2}}}$ on $\Omega(h) \cap\left\{|\operatorname{Re} z-E(h)|>4 h^{k}\right\}$ for some constant $C>0$, where $\alpha=S(h) h^{-n^{\sharp}-1}$.

In fact, take any function $\psi_{h} \in C_{0}^{\infty}(\mathbb{R})$ such that $0 \leq \psi_{h} \leq 1$ and

$$
\psi_{h}(x)=\left\{\begin{array}{l}
0 \text { when }|x-E(h)| \geq 3 h^{k} \\
1 \text { when }|x-E(h)| \leq 2 h^{k}
\end{array}\right.
$$

Let

$$
f(z, h)=\left(\pi \alpha^{2}\right)^{-1 / 2} \int \exp \left(-\frac{(x-z)^{2}}{\alpha^{2}}\right) \psi_{h}(x) d x, \quad \alpha=S(h) h^{-n^{\sharp}-1},
$$

Note that $\left(\pi \alpha^{2}\right)^{-1 / 2} \int \exp \left(-(x-z)^{2} / \alpha^{2}\right) d x=1$. Then

(1) Clearly $f(z, h)$ is holomorphic in $\mathbb{C}$. 
(2) For $z \in \Omega(h)$, write $z=u+i v$, we have

$$
\begin{aligned}
|f(z, h)| & \leq\left(\pi \alpha^{2}\right)^{-1 / 2} \int\left|\exp \left(-\frac{(x-z)^{2}}{\alpha^{2}}\right) \psi_{h}(x)\right| d x \\
& \leq\left(\pi \alpha^{2}\right)^{-1 / 2} \exp \left(\frac{v^{2}}{\alpha^{2}}\right) \int \exp \left(-\frac{(x-u)^{2}}{\alpha^{2}}\right) d x \\
& =\exp \left(\frac{v^{2}}{\alpha^{2}}\right) \leq e .
\end{aligned}
$$

(3) For $z \in\left[E(h)-h^{k}, E(h)+h^{k}\right]$, we have

$$
\begin{aligned}
|f(z, h)-1| & \leq\left(\pi \alpha^{2}\right)^{-1 / 2} \int \exp \left(-\frac{(x-u)^{2}}{\alpha^{2}}\right)\left|\psi_{h}(x)-1\right| d x \\
& \leq \pi^{-1 / 2} \int e^{-y^{2}}\left|\psi_{h}(\alpha y+u)-1\right| d y \\
& \leq \pi^{-1 / 2} \int_{\mathbb{R} \backslash B\left(0, \frac{h^{k}}{\alpha}\right)} e^{-y^{2}} d y \\
& <\frac{1}{2}
\end{aligned}
$$

when $h$ is sufficiently small since $\frac{h^{k}}{\alpha} \rightarrow \infty$ as $h \rightarrow 0$. ( Recall $S(h)=$ $\mathcal{O}\left(h^{\infty}\right)$ and so is $\left.\alpha\right)$

(4) For $z \in \Omega(h) \cap\left\{|\operatorname{Re} z-E(h)|>4 h^{k}\right\}$, we have

$$
\begin{aligned}
|f(z, h)| & \leq\left(\pi \alpha^{2}\right)^{-1 / 2} \exp \left(\frac{v^{2}}{\alpha^{2}}\right) \int \exp \left(-\frac{(x-u)^{2}}{\alpha^{2}}\right) \psi_{h}(x) d x \\
& \leq\left(\pi \alpha^{2}\right)^{-1 / 2} \exp \left(\frac{v^{2}}{\alpha^{2}}\right) \int_{B\left(E(h), 3 h^{k}\right)} \exp \left(-\frac{h^{2 k}}{\alpha^{2}}\right) d x \\
& \leq C e^{-C \frac{h^{2 k}}{\alpha^{2}}} \text { for some constant } C>0,
\end{aligned}
$$

when $h$ is sufficiently small.

The second step is an application of the maximum principle in the domain $\Omega(h)$ - see Fig.2. For that let us put $G(z, h)=e^{-i z \sigma} f(z, h) F(z, h)$ where

$$
\sigma=\frac{\log \left(S(h) A \exp \left(A h^{-n^{\sharp}} \log \frac{1}{h S(h)}\right)\right)}{S(h)\left(1+h^{-n^{\sharp}-1}\right)} .
$$

Then $G(z, h)$ is a holomorphic function on $\Omega(h)$. For $h \in\left\{h_{j}\right\}_{j \geq 1}$ and $h<\tilde{h}$, we have

On $\left[E(h)-5 h^{k}, E(h)+5 h^{k}\right]+i S(h)$ 


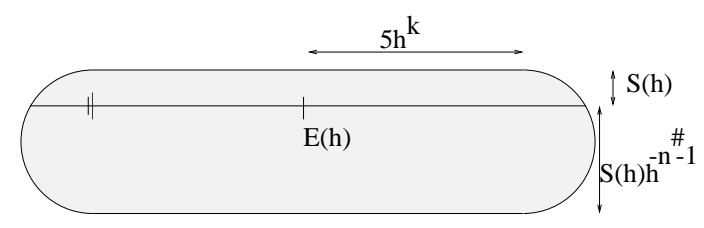

FIgURE 2. The domain in which the maximum principle is applied

$$
\begin{aligned}
|G(z, h)| & \leq\left|e^{-i z \sigma}\|f(z, h)\| F(z, h)\right| \\
& \leq e^{S(h) \sigma} e \frac{1}{S(h)} .
\end{aligned}
$$

On $\left[E(h)-5 h^{k}, E(h)+5 h^{k}\right]-i S(h) h^{-n^{\sharp}-1}$

$$
\begin{aligned}
|G(z, h)| & \leq e^{-S(h) h^{-n^{\sharp}-1} \sigma} e A \exp \left(A\left(\log \frac{1}{h S(h)}\right) h^{-n^{\sharp}}\right) \\
& =e^{S(h) \sigma} e \frac{1}{S(h)} \quad \text { by the choice of } \sigma .
\end{aligned}
$$

On $\left(E(h) \pm 5 h^{k}\right)+i\left[-S(h) h^{-n^{\sharp}-1}, S(h)\right]$

$$
\begin{aligned}
|G(z, h)| & \leq e^{S(h) \sigma} C \exp \left(-C \frac{h^{2 k}}{\alpha^{2}}\right) A \exp \left(A\left(\log \frac{1}{h S(h)}\right) h^{-n^{\sharp}}\right) \\
& \leq \mathcal{O}(1) e^{S(h) \sigma}<e^{S(h) \sigma} \frac{1}{S(h)},
\end{aligned}
$$

when $h$ is sufficiently small.

Hence applying the maximum principle, we have

$$
\begin{aligned}
\max _{\Omega(h)}|G(z, h)| & \leq e^{S(h) \sigma} e \frac{1}{S(h)} \\
& =\mathcal{O}(1) \frac{1}{S(h)}
\end{aligned}
$$

when $h$ is sufficiently small.

Thus, there exists $h(S)>0$ and $B>0$ such that $\max _{\Omega(h)}|G(z, h)| \leq \frac{B}{S(h)}$ when $h \in\left\{h_{j}\right\}_{j \geq 1}$ and $h<h(S)$. Hence,

$$
\begin{aligned}
\frac{B}{S(h)} & \geq \max _{z \in\left[E(h)-h^{k}, E(h)+h^{k}\right]}\left|e^{i z \sigma}\right||f(z, h)||F(z, h)| \\
& \geq \frac{1}{2} \max _{z \in\left[E(h)-h^{k}, E(h)+h^{k}\right]}|F(z, h)|,
\end{aligned}
$$

which implies

$$
|F(z, h)| \leq \frac{2 B}{S(h)}, \quad \forall z \in\left[E(h)-h^{k}, E(h)+h^{k}\right] .
$$


This completes the proof of Lemma 2 .

\section{Acknowledgements}

This paper was written while the authors were visiting the Fields Institute and they would like to thank the Institute for its hospitality. Thanks go also to Antônio Sá Barreto for his generous help during the preparation of this paper and to Shu Nakamura and Georgi Popov for helpful discussions.

\section{References}

1. V. M. Babich and V. S. Buldyrev, Short-wavelength diffraction theory. Asymptotic methods. Translated from the 1972 Russian original by E. F. Kuester. Springer Series on Wave Phenomena, 4, Springer-Verlag, Berlin, 1991.

2. Y. Colin de Verdière, Quasimodes sur les Variétés Riemannienes, Invent. Math. 43 (1977), $15-52$.

3. L. Guillopé and M. Zworski, Scattering asymptotics for Riemann surfaces, Ann. of Math. 145 (1997), 597-660.

4. V. F. Lazutkin, KAM theory and semiclassical approximations to eigenfunctions, With an addendum by A. I. Shnirelman. Ergebnisse der Mathematik und ihrer Grenzgebiete 24, Springer-Verlag, Berlin, 1993.

5. R. B. Melrose, Polynomial bounds on the distribution of poles in scattering by an obstacle, Journées "Équations aux Dérivées Partielles", Saint-Jean-de-Monts, 1984.

6. G. Popov, Quasi-modes for the Laplace operator and glancing hypersurfaces in Proceedings of Conference on Microlocal Analysis and Nonlinear Waves, Minnesota 1989, M. Beals, R. Melrose and J. Rauch eds. Springer Verlag, Berlin-Heidelberg-New York, 1991.

7. G. Popov and G. Vodev, Existence of resonances in the transmission problem, preprint, 1997.

8. J. Ralston, Approximate Eigenfunctions of the Laplacian, J. Diff. Geom. 12 (1977), 87100.

9. J. Sjöstrand, A trace formula and review of some estimates for resonances, in Microlocal analysis and spectral theory (Lucca, 1996), 377-437, NATO Adv. Sci. Inst. Ser. C Math. Phys. Sci., 490, Kluwer Acad. Publ., Dordrecht, 1997.

10. J. Sjöstrand and M. Zworski, Complex scaling and the distribution of scattering poles, Jour. Amer. Math. Soc., 4 (1991), 729-769

11. P. Stefanov and G. Vodev, Distribution of resonances for the Neumann problem in linear elasticity outside a strictly convex body, Duke Math. J. 78 (1995), 677-714

12. _ Neumann resonances in linear elasticity for an arbitrary body, Comm. Math. Phys. 176 (1996), 645-659.

13. G. Vodev, Sharp Polynomial Bounds on the Number of Scattering Poles for Perturbations of the Laplacian, Comm. Math. Phys. 146 (1992), 39-49.

14. M. Zworski, unpublished, 1990.

15. _ Poisson formula for resonances, Séminaire E. D. P. 1996-1997, École Polytechnique, XIII-1-XIII-12.

Dept. of Math., Purdue University, West Lafayette, IN 47907-1399

E-mail address: stang@math.purdue.edu

Dept. of Math., University of Toronto, Toronto, On, CANADA M5S 1A4

E-mail address: zworski@math.toronto.edu 